\title{
Asymptomatic and Symptomatic Carotid Stenosis: An Obsolete Classification?
}

\author{
Anastasios Chatzikonstantinou, Marc E. Wolf, Anke Schaefer, and Michael G. Hennerici
}

Department of Neurology, UniversitätsMedizin Mannheim, University of Heidelberg, Theodor-Kutzer-Ufer 1-3, 68167 Mannheim, Germany

Correspondence should be addressed to Anastasios Chatzikonstantinou, chatziko@neuro.ma.uni-heidelberg.de

Received 28 July 2011; Accepted 16 October 2011

Academic Editor: Tatjana Rundek

Copyright ( $(2012$ Anastasios Chatzikonstantinou et al. This is an open access article distributed under the Creative Commons Attribution License, which permits unrestricted use, distribution, and reproduction in any medium, provided the original work is properly cited.

\begin{abstract}
Since many years, clinical decisions about the management of patients with carotid stenosis have been based on the distinction between "asymptomatic" and "symptomatic" presentations. This was also reflected by the design of previous studies on the surgical versus conservative treatment and of current studies on interventional treatment versus surgery. Both terms, however, only address different phases of activity of the one and the same condition and blur the significant message that carotid stenosis is a most important marker of systemic atherosclerosis, which is accompanied by a much higher risk of cardiovascular events rather than stroke. As a consequence, early diagnosis and followup during best medical treatment, life-style management, regular cardiovascular assessment, and good control of all vascular risk factors should be recommended in all patients with carotid stenosis-whether identified in the long-lasting "silent" or short-lasting "vulnerable" period lasting only a few weeks after cerebral ischemia. Patients in this short time window benefit from additional carotid intervention, under the condition of an individually favorable benefit-risk ratio ("individual vulnerability").
\end{abstract}

\section{Introduction}

Carotid stenosis is common, especially in patients with vascular risk factors or with coexistent pathology of coronary or peripheral arteries $[1,2]$. Since many decades, the classification between "symptomatic" and "asymptomatic" carotid stenosis has dominated the management of affected patients. This distinction corresponds to the design and results of previous clinical trials on surgical versus medical treatment of carotid stenosis for stroke prevention as well as of current studies comparing surgical with interventional procedures (angioplasty with or without stent) [3-8].

However, careful review of these studies, as well as longterm natural history observations for many decades, shows that carotid stenosis is much more sensitive as marker of systemic atherosclerosis than a cause of stroke $[9,10]$. Only within a small time window, when carotid stenosis shows progression and high plaque vulnerability, either in the presence of or without clinical signs or symptoms, there is a higher incidence of stroke.
First descriptions of carotid stenosis related to cerebrovascular events date back to T. Willis (1621-1675) and J. J. Wepfer (1620-1695). However, it was not before the 1950s when surgical interventions in the acute phase of stroke or for secondary stroke prevention were reported by DeBakey and Eastcott $[11,12]$.

In the 1980s, with the introduction of vascular ultrasound, the true dimensions of carotid disease became apparent. Quite unsurprisingly, a high number of patients were diagnosed with a so-called "asymptomatic" carotid stenosis [2], but the prognosis and management of these patients were totally uncertain. Studies performed since then showed already very early an eminent contrast between a very low incidence of stroke (1-2\%/year) and a rather high cardiovascular morbidity and mortality (5-10\%/year), in particular in patients with clinically silent presentation (socalled "asymptomatic carotid disease") $[9,10]$. The same is true for the "symptomatic" carotid stenosis too, but with one difference: there is a higher risk of stroke (10-20\%) within the first 14-28 days following a cerebrovascular event (TIA 
or stroke) [13]. After this vulnerable period, the stroke risk declines to that of the "asymptomatic" carotid stenosis.

\section{Pathophysiology of Carotid Stenosis}

In most cases, carotid stenosis is the result of atherosclerotic changes of the vessel wall. Other causes (e.g., dissection, often observed in patients under 55 years of age) only account for a small fraction of carotid lesions.

Atherosclerosis is a progressive disease with periods of stability and sometimes reparation, which begins with endothelial damage of the vessel wall already in infancy and adolescence. There are many factors promoting this damage: arterial hypertension, elevated blood lipid levels and problems of cholesterol or glucose metabolism, reduced release of nitrogen oxide, but also genetic components such as expression of vascular cell adhesion molecules (VCAM1) [14-19]. Functional disturbance of laminar blood flow, particularly in arterial junctions, promotes the accumulation of LDL and consecutively lead to remodeling of the vessel wall. Monocyte migration and adhesion in the region of endothelial damage then follows, induced by proinflammatory cytokines [20, 21]. Identification of these mechanisms as biomarkers for the activity of atherosclerosis has confirmed the association of general risk factors with vascular degeneration but unfortunately did not contribute much to the sensitivity/specificity of a biomarker to predict the individual stroke risk in a "vulnerable patient."

\section{Diagnostic Methods}

Significant improvements in diagnostic methods used for detection of carotid stenosis have influenced our knowledge about causes, spontaneous course, and risks of this disease. Initially, auscultation of carotid bruits was the gold standard in clinical practice. Systematic evaluation of this method, however, revealed a very low specificity and sensitivity [2]. Digital subtraction angiography (DSA) was for a long time the only method able to reliably identify a carotid stenosis, but it was only used in preselected patients because of its invasive nature and the risks associated with the procedure. It was the introduction of vascular ultrasound, with its noninvasive nature and good visualization, that made better carotid screening possible in a large number of patients, including many in clinically silent periods. Nowadays, highly developed ultrasound systems allow visualization of vessel wall changes in early stages, various grades of vessel stenosis and of intraluminal flow phenomena, as well as analysis of plaque texture and structure in real time and $3 \mathrm{D}$ or even $4 \mathrm{D}$ [22]. An experienced user can perform a highly sophisticated and reliable examination, which can be repeated during long-term monitoring without any risks or complications for the patient. Such technologies have recently been introduced in large clinical trials (e.g., PERFORM study) [23]. Ultrasound imaging can be used for screening and control of intima-media thickness (IMT), plaque morphology, grade and progression in clinical practice, as well as in clinical and epidemiological studies $[24,25]$. Today, DSA is only rarely necessary—as opposed to the time when NASCET [3], ECST [4] and ACAS [5] were carried out. In addition to ultrasound, examinations such as CT and MR angiography allow collection of supplementary diagnostic insights, for example, perfusion studies, detection of vessel anomalies (aneurysms, angiomas), and plaque imaging, and can also be useful in cases where vessels are not well accessible via ultrasound or as an alternative investigation [26, 27].

Despite these technical approaches and changes in diagnostic practice, the current classification of the degree of stenosis still corresponds to the modalities of angiographic measurements used in the aforementioned clinical trials, because they formed the basis for evidence-based regimens. However, the information provided by ultrasound, CTA, and MRA today is by far better for an individual decision than the restricted use of only three stages of obstruction: high grade $(>75 \%)$, moderate $(60-75 \%)$, and low grade $(<50 \%$ of local lumen reduction). If combined with other parameters such as individual clinical presentation ("the vulnerable patient"), modern brain imaging, and plaque structure analysis ("the vulnerable plaque"), a best estimation of the individual stroke risk associated with carotid disease can be made regarding the therapeutic consequences. These parameters allow a differentiation between "active" (vulnerable plaques) and "stable" disease. Echomorphologic features (plaque ulcerations, hemorrhage, lipid accumulation), which can be identified through high-definition ultrasound imaging and/or application of contrast agent, correlate with histopathologic characteristics and signalize a high risk for arterioarterial embolization [28]. HITS (high-intensity transient signals) registered over the middle cerebral artery indicate microembolization and can facilitate the decision of individual treatment strategies with suitable medication (CARESS [29]), as can detection of progressive carotid stenosis or silent infarcts in cerebral CT/MRT be interpreted as a sign of activity or acuity of a carotid stenosis [30-32].

\section{Studies}

Most of our knowledge on the topic of carotid disease, especially regarding treatment and course of the disease, was derived from studies done in the last 25 years. Some studied the natural history and reported clinical and vascular followup, as did Chambers and Norris (1986) [9] and Hennerici et al. (1987) [10].

As far as the so-called "symptomatic" carotid stenosis is concerned, two major, multicenter, randomized controlled studies are widely known: the North American Symptomatic Carotid Endarterectomy Trial (NASCET) with 2885 patients and the European Carotid Surgery Trial (ECST) with 3024 patients $[3,4]$.

The two randomized controlled studies mostly referenced on the subject of "asymptomatic" carotid stenosis are the Asymptomatic Carotid Atherosclerosis Study (ACAS) with 1662 and the Asymptomatic Carotid Surgery Trial (ACST) with 3120 patients $[5,6]$.

Table 1 summarizes the most important results of the aforementioned studies [3-6, 33-38]. 
TABLE 1: Outline of the most important characteristics and results of large carotid surgery trials. DSA: digital substraction angiography.

\begin{tabular}{|c|c|c|c|c|c|c|}
\hline & \multicolumn{2}{|c|}{ NASCET } & \multicolumn{2}{|c|}{ ECST } & ACAS & ACST \\
\hline$n$ (total) & \multicolumn{2}{|c|}{2885} & \multicolumn{2}{|c|}{3024} & 1662 & 3120 \\
\hline $\begin{array}{l}\text { Observation period prior to inclusion } \\
\text { (max.) }\end{array}$ & \multicolumn{2}{|c|}{120 days } & \multicolumn{2}{|c|}{180 days } & 120 days & 180 days \\
\hline $\begin{array}{l}\text { Ratio TIA/stroke as qualifying event } \\
\text { prior to inclusion }\end{array}$ & \multicolumn{2}{|c|}{$61 \% / 39 \%$} & \multicolumn{2}{|c|}{$50 \% / 50 \%$} & - & - \\
\hline $\begin{array}{l}\text { Diagnostic method for determination } \\
\text { of stenosis grade }(\mathrm{SG})\end{array}$ & \multicolumn{2}{|c|}{ DSA (distal SG) } & \multicolumn{2}{|c|}{ DSA (local SG) } & $\begin{array}{c}\text { Doppler } \\
\text { sonography/DSA }\end{array}$ & $\begin{array}{l}\text { Duplex } \\
\text { sonography }\end{array}$ \\
\hline $\begin{array}{l}\text { Significant correlation of stroke risk } \\
\text { with SG }\end{array}$ & \multicolumn{2}{|c|}{ Yes } & \multicolumn{2}{|c|}{ Yes } & No & No \\
\hline Controlled medical treatment & \multicolumn{2}{|c|}{ No } & \multicolumn{2}{|c|}{ No } & No & No \\
\hline Indication for carotid surgery & $\begin{array}{l}\text { Limited at } \\
50-69 \% \text { SG }\end{array}$ & $>70-99 \%$ SG & $\begin{array}{l}\text { Limited at } \\
50-69 \% \text { SG }\end{array}$ & $>70-99 \%$ SG & No & $\begin{array}{l}\text { Limited at } \\
>60-99 \%\end{array}$ \\
\hline Stroke risk (surgical treatment) (\%) & 15.7 (5 yrs) & 8.9 (3 yrs) & $15.0(5 \mathrm{yrs})$ & 10.5 (5 yrs) & $5.1(5 \mathrm{yrs})$ & 6.4 (5 yrs) \\
\hline Stroke risk (medical treatment) (\%) & $22.2(5 \mathrm{yrs})$ & 28.3 (3 yrs) & 12.1 ( 5 yrs $)$ & 19.0 (5 yrs) & $11.0(5 \mathrm{yrs})$ & 11.7 (5 yrs) \\
\hline Absolute risk reduction (\%) & 6.5 & 19.4 & -2.9 & 8.5 & 5.9 & 5.4 \\
\hline Relative risk reduction (\%) & 29 & 69 & - & 45 & 53 & 46 \\
\hline Numbers needed to treat & 15 & 5 & - & 12 & 17 & 19 \\
\hline
\end{tabular}

There is also a plethora of meta-analysis data available in the literature $[34,36-38]$.

\section{5. "Symptomatic" Carotid Stenosis}

In NASCET, the annual stroke rate under uncontrolled medical treatment (within 2 years of followup) was $13 \%$ for high-grade stenosis ( $>70 \%$ distal degree of stenosis) and about $7 \%$ for moderate stenosis. Carotid occlusions were found to be associated with a low risk of stroke (2.1\%/year for ipsilateral and 5.5\%/year for all strokes) [3]. Therefore, carotid endarterectomy was not recommended for low degree of stenosis, as well as for patients of subtotal carotid stenosis ("pseudoocclusion"). The annual stroke rate in ECST was up to $7 \%$ for high-grade stenosis ( $>90 \%$ local degree of stenosis) in the-also uncontrolled-medical treatment branch within a 3-year followup and as low as $1.5 \%$ for $70-89 \%$ local degree of stenosis [36, 39]. Both studies recruited patients with a qualifying event (stroke, TIA or retinal TIA, the latter being the case in about $1 / 3$ of NASCET patients) within a time window of 4 to 6 months prior to recruitment. Many of these cerebrovascular events were not evaluated with modern brain imaging (CT or $\mathrm{MRI}$ ), and the association with the carotid stenosis was made only based on patients' histories. Other coincident potential sources of stroke or TIA, such as lacunar infarcts due to small vessel disease or cardioembolic infarcts from atrial fibrillation, were not taken into consideration [40], which is a major drawback compared to modern clinical workup.

The results of both studies were interpreted as a significant advantage of carotid surgery over medical treatment, which mainly consisted of acetylsalicylic acid, but lacked systematic, prospective study design of other risk factor management and monitoring of compliance during the study.

Data in the literature indicates that relevant carotid stenosis $(>75 \%)$ is the underlying cause in only $5-12 \%$ of all cerebral ischemic events [3, 4]: about $20 \%$ of strokes in the territory of a "symptomatic" carotid stenosis cannot be without any doubt attributed to carotid stenosis, for example, in case of coexisting lacunar or cardioembolic causes [41].

The new ASCO stroke subtype classification [42] is suited to display such coexisting stroke causes. We prospectively studied 158 consecutive patients ( 89 men and 69 women, mean age $75 \pm 11$ years) with carotid stenosis $\geq 50 \%$ (as diagnosed on Doppler/Duplex vascular imaging) admitted to our stroke unit in 2010 with the diagnosis of ischemic stroke $(142 ; 89.9 \%)$ or TIA $(16 ; 10.1 \%)$, based on clinical features and brain imaging (CT/MRI). Patients' characteristics, risk factors, and the distribution of the grades of the 232 carotid stenosis detected are displayed in Table 2. The ASCO score (A: atherothrombosis; S: small vessel disease; C: cardioembolism; $\mathrm{O}$ : other causes with corresponding grades: (1) definitely a potential cause, (2) causality uncertain, (3) disease present, but unlikely a cause, (0) no disease present, and (9) no suitable tests performed) was used to classify stroke etiology after a thorough stroke workup was completed.

As determined by ASCO (Figure 1), carotid stenosis was the probable cause of the ischemic event only in less than half of the patients recruited (grade A1; 65; 41.1\%). However, in 17 patients $(10.8 \%)$ this was combined with other equally probable stroke causes $(2 ; 3.1 \% \mathrm{~S} 1$ and $15 ; 9.5 \% \mathrm{C} 1)$. Interestingly, only if degrees of stenosis $\geq 70 \%(78 ; 49.3 \%)$ were considered, the majority of patients were identified correctly. However, there were still 15 patients with A1 and 


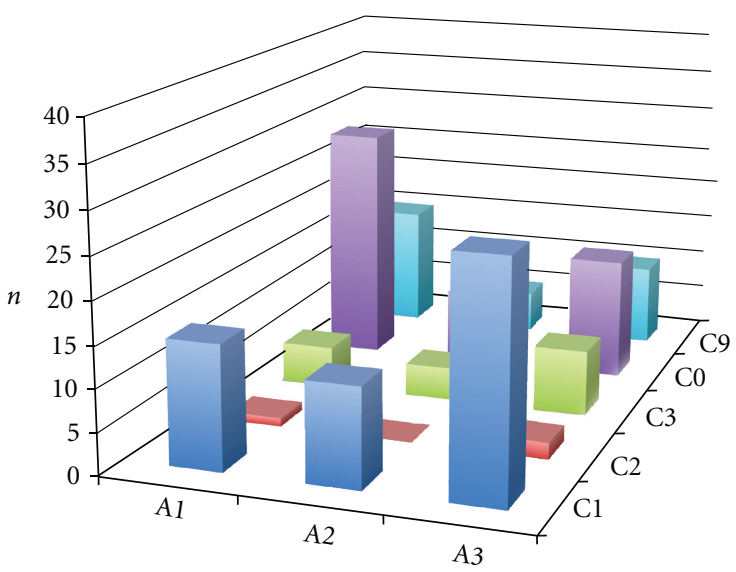

(a)

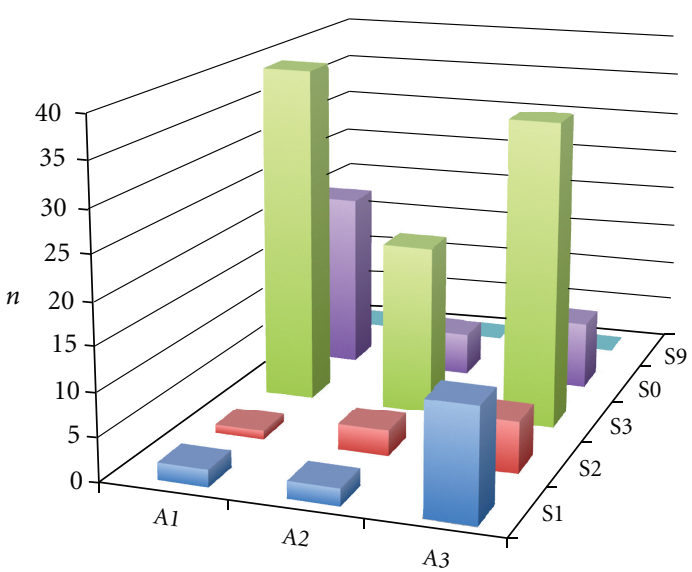

(b)

FIGURE 1: ASCO classification of stroke etiologies. The ASCO classification for "A" (atherothrombosis), "S" (small vessel disease), and "C" (cardioembolic). The "O" (other causes) group was omitted because only 4 patients received a grade other than "0." As all patients had Duplex/Doppler imaging and carotid stenosis, no patient had "A0" or "A9," so these groups were omitted. The figure visualizes competing/coexisting etiologies.

TABle 2: Patient characteristics.

\begin{tabular}{|c|c|c|}
\hline Parameter & Value & $\%$ \\
\hline Total of patients & 158 & 100 \\
\hline Men & 89 & 56.3 \\
\hline Women & 69 & 43.7 \\
\hline Mean age $( \pm$ SD $)$ & $75 \pm 11$ & - \\
\hline Cerebral ischemia & 142 & 89.9 \\
\hline TIA & 16 & 10.1 \\
\hline Unilateral stenosis & 84 & 53.2 \\
\hline Bilateral stenosis & 74 & 46.8 \\
\hline \multicolumn{3}{|l|}{ Carotid stenosis grades } \\
\hline Carotid stenosis in total & 232 & \\
\hline$\geq 50-59 \%$ & 74 & $(31.9)$ \\
\hline$\geq 60-69 \%$ & 68 & $(29.3)$ \\
\hline$\geq 70-79 \%$ & 24 & $(10.3)$ \\
\hline$\geq 80-89 \%$ & 28 & $(12.1)$ \\
\hline$\geq 90-99 \%$ & 29 & $(12.5)$ \\
\hline $100 \%$ & 9 & $(3.9)$ \\
\hline \multicolumn{3}{|l|}{ Concomitant vascular diseases } \\
\hline Coronary artery disease & 38 & $24.1^{*}$ \\
\hline Peripheral artery disease & 18 & 11.4 \\
\hline \multicolumn{3}{|l|}{ Vascular risk factors } \\
\hline Arterial hypertension & 140 & 88.6 \\
\hline Hyperlipidemia & 77 & 48.7 \\
\hline Diabetes mellitus & 54 & 34.2 \\
\hline
\end{tabular}

${ }^{*}$ Correlation with stenosis grade: $P<0.05$.

C1 (9.5\%). Cardioembolism was the most probable stroke cause in $55(34.8 \%)$ and small vessel disease in $17(10.8 \%)$ patients. Hence, 93 patients $(58.9 \%)$ had an "asymptomatic" carotid stenosis despite suggested acute stroke from carotid stenosis.

\section{6. "Asymptomatic" Carotid Stenosis}

Both natural history studies, as well as several large, multicenter, randomized controlled studies, have shown that "asymptomatic" carotid stenosis is quite a benign disease, with an annual stroke rate of only 1-2\% and a stroke-related annual mortality rate of merely $<0.05 \%[5,10]$.

Carotid stenosis, however, is an established risk factor for myocardial infarction. The annual mortality, mainly because of cardiovascular events, is about $6-8 \%$. About $40 \%$ of patients with "asymptomatic" carotid stenosis have coincident coronary artery disease (CAD) [10, 43, 44]. In many patients, silent atherosclerotic alterations of coronary vessels lead to a significantly increased mortality. ACAS showed a mortality rate of $6 \%$ with one year of followup [5]. The highest prevalence $(28 \%)$ of carotid stenosis was found in patients with peripheral artery disease (PAD); these patients also have the highest mortality [2].

In our patient group described above, about one-fourth of the patients $(38 ; 24.1 \%)$ had CAD and 18 (11.4\%) PAD. There was even a statistically significant correlation between grade of stenosis and presence of CAD $(P=0.018)$. Classic vascular risk factors were very common: arterial hypertension $(140 ; 88.6 \%)$, hyperlipidemia $(77 ; 48.7 \%)$, and diabetes mellitus $(54 ; 34.2 \%)$.

ACAS and ACST could show a minor preventive effect of carotid surgery in carotid stenosis between $60 \%$ and $99 \%$. A refined assessment of risk according to stenosis grade could not be made because of various methodological problems. In ACAS, absolute risk reduction of stroke and/or death was $5.9 \%$ and in ACST $5.4 \%$, in a time frame of 5 years. However, it should be taken into consideration 
TABLE 3: Factors that increase stroke risk in patients with carotid stenosis.

\begin{tabular}{l}
\hline Acute hemispheric ischemia associated with carotid stenosis in \\
the last 4 weeks \\
Ipsilateral silent infarcts in CT/MRI \\
Intracranial artery stenosis \\
Contralateral carotid occlusion \\
Insufficient medical treatment (risk factors) \\
Insufficient collateralisation over the circulus Willisii \\
Coexistent coronary/peripheral artery disease \\
Leukoaraiosis \\
HITS detection \\
Rapid progression of carotid stenosis \\
Plaque ulcerations \\
Highly echolucent plaques in carotid duplexsonography \\
High Lp-PLA $\mathrm{A}_{2}$ Concentration
\end{tabular}

that the perioperative complication risk in both studies was extremely low $(2.3 \%$ and $2.9 \%)$, because of a very strict selection of surgeons $[5,6]$. Nevertheless, many patients were treated unnecessarily because of the large number needed to treat (17-19).

Control of risk factors was reported in ACST on a retrospective analysis suggesting that the already small benefit of surgery was minimized or lost if adequate monitoring of risk factor management had been achieved $[6,45]$.

Results from studies with very long followups from our department support these findings of low stroke mortality rate in these patients $[10,46]$.

\section{New Assessment of Prognosis and Management}

Based on the available data [47-51], a number of factors associated with a high risk of stroke on the basis of a carotid stenosis can be identified (Table 3 ).

Associated with low risk of stroke are Retinal TIA, no (or few) vascular risk factors, effective intracranial collateralisation over the circulus Willisii, as well as subtotal stenosis [52-54].

Indications of an unstable ("vulnerable") plaque are plaque ulcerations, high concentration of lipoproteinassociated phospholipase $\mathrm{A}_{2}$ (Lp-PLA $\mathrm{A}_{2}$ ), and highly echolucent plaques [55-59].

Beside the simple clinical risk score $\mathrm{ABCD}^{2}$ (age, blood pressure, clinical features, duration, diabetes) [60], the risk model proposed by Rothwell can help to assess the individual risk for ipsilateral cerebrovascular events in patients with carotid stenosis [54, 61]. It is based on meta-analysis data of the major carotid studies (particularly the ECST) and may support in making an individual treatment decision (Table 4).

\section{Conclusions}

Uncritical usage of the results from the aforementioned studies often misguides and leads to false conclusions, generalizations, and uncertainty about best available modern treatment. This problem rises at least partly from the different methods used to evaluate the stenosis grade (angiography versus ultrasound) and from the nonvalidated evaluation criteria. Therefore, ACAS and ACST could not show any connection between stenosis grade and stroke risk, whereas this connection was clearly shown not only in natural history studies but also in NASCET and ECST. Because patients nowadays do not usually undergo conventional angiography before carotid surgery, the direct justification for surgery by these studies' results is actually missing.

Other problems and insecurities in the management of carotid stenosis result from the fact that a significantly positive effect of carotid surgery (in NASCET and ECST) was shown only in patients who underwent surgery within 2-4 weeks, but not in those operated upon later, up to 46 months. Furthermore, there are significant asymmetries in the recruitment of men/women in all 4 major studies. These asymmetries were not corrected in the analysis of the primary endpoints, thus undermining the results of ACAS and ACST in particular [62]. Possible overlapping of different stroke pathogenesis was not taken into consideration, which limits the direct transfer of study results to the individual patient in everyday clinical practice. In our patient collective, for example, carotid stenosis was the probable cause of stroke in less than half of patients and even in part of these patients there was an overlapping with other (mostly cardiac) causes. The ASCO score is especially suitable to demonstrate overlapping stroke etiologies and to quickly create a rudimental risk profile of the patient [42]. Often lacking a sufficient brain imaging (especially MRI) and thorough stroke workup, as it is performed today before every carotid surgery, the studies failed to take some of these factors into account. Finally, adequate medical management with monitoring and controlled treatment of vascular risk factors (e.g., statin and/or antihypertensive treatment) was missed.

A strict classification in "symptomatic" and "asymptomatic" carotid stenosis and decisions on the degree of carotid stenosis alone could therefore be misleading. Carotid disease should be rather regarded as an entity with active and stable phases. When deciding about treatment, one should differentiate between those two phases utilizing clinical findings, followups (e.g., rapid progression, repeated TIAs), or other parameters (silent infarcts in brain imaging, HITS, plaque configuration). During an active phase, carotid surgery is advisable under the condition that it is performed early and that the perioperative complication risk is low $(<5 \%)[47,63]$. Carotid angioplasty with or without stent is currently not a routine option but can be performed by highly experienced interventionalists in selected patients who cannot undergo a surgery. None of the major studies 
TABLE 4: Risk model by Rothwell for ipsilateral territorial infarcts in all patients in the medical treatment branch of ECST [61]. CI: confidence interval.

\begin{tabular}{lccc}
\hline & $P$ & Hazard ratio & 95\% CI \\
\hline Cerebral events versus ocular events & 0.008 & 2.45 & $1.27-4.75$ \\
Residual neurological signs after 7 days & 0.006 & 1.82 & $1.08-1.57$ \\
Diabetes & 0.007 & 1.71 & $1.18-2.80$ \\
Any ischemic event within the last 2 months & 0.003 & 1.02 & $1.20-2.44$ \\
Number of events within the last 3 months (per event) & 0.01 & 1.31 & $1.01-1.03$ \\
Previous myocardial infarction & 0.02 & 1.34 & $1.04-1.65$ \\
Degree of carotid stenosis & 0.0000 & 1.80 & $1.30-1.38$ \\
Plaque surface irregularity & 0.01 & 0.40 & $1.14-2.83$ \\
Poststenotic collapse of the internal carotid artery & 0.03 & 1.01 & $0.17-0.94$ \\
Age (per year) & 0.62 & 1.23 & $0.98-1.03$ \\
Male sex & 0.31 & 1.05 & $0.83-1.82$ \\
Systolic blood pressure (per 10 mmHg) & 0.82 & 1.10 & $0.90-1.15$ \\
Diastolic blood pressure (per 10 mmHg) & 0.61 & 1.03 & $0.80-1.30$ \\
Peripheral vascular disease & 0.90 & 0.96 & $0.65-1.63$ \\
Angina without previous myocardial infarction & 0.77 & 0.90 & $0.71-1.29$ \\
ECG signs of left ventricular hypertrophy & 0.18 & 0.96 & 1.32 \\
Cerebral infarct on symptomatic side on CT & & 1.00 & $0.40-2.10$ \\
Occlusion of the contralateral internal carotid artery & & $0.72-1.63$ & \\
\hline
\end{tabular}

until today (SPACE, EVA3S, CAVATAS, ICSS, CREST) could show a noninferiority of angioplasty compared with carotid surgery $[64,65]$.

During a stable phase, the patient with carotid stenosis is a patient in high risk for cardiovascular events. In accordance with results from previous studies [10, 43, 44], our patients had a high percentage of concomitant vascular diseases and risk factors. Almost all patients had an arterial hypertension, and hyperlipidemia and diabetes were also very common. Furthermore, PAD and CAD were quite common, and the presence of $\mathrm{CAD}$ even correlated with the grade of carotid stenosis. The risks derived from these diseases are not diminished by a surgical or interventional treatment of the carotid stenosis [52]. Because of the high cardiovascular risk, however, these patients do profit from controlled medical treatment. Every risk factor increases vascular risk by its own, so there is a summation effect of their combined treatment on the prognosis [66]. Particularly under statin treatment, it could be shown that not only there is a reduction of TIA/stroke and of cardiovascular morbidity and mortality associated with coincidental coronary heart disease but also a decrease in the necessity of vascular interventions (56\% of relative risk reduction) [67]. SPACE2 will possibly clarify if there is need of an interventional carotid treatment in these cases. Other cerebrovascular risk factors, such as atrial fibrillation, should certainly be also taken into consideration. Conservative treatment strategies and duplex sonographic followups (in most cases every 3-6 months) are essential in order to detect a phase of activity in time.

\section{References}

[1] J. S. Fine-Edelstein, P. A. Wolf, D. H. O’Leary et al., "Precursors of extracranial carotid atherosclerosis in the Framingham Study," Neurology, vol. 44, no. 6, pp. 1046-1050, 1994.

[2] M. Hennerici, A. Aulich, W. Sandmann, and H. J. Freund, "Incidence of asymptomatic extracranial arterial disease," Stroke, vol. 12, no. 6, pp. 750-758, 1981.

[3] "North American Symptomatic Carotid Endarterectomy Trial Collaborators: beneficial effect of carotid endarterectomy in symptomatic patients with high-grade carotid stenosis," New England Journal of Medicine, vol. 325, no. 7, pp. 445-453, 1991.

[4] "Randomised trial of endarterectomy for recently symptomatic carotid stenosis: final results of the MRC European Carotid Surgery Trial (ECST)," Lancet, vol. 351, no. 9113, pp. 1379-1387, 1998.

[5] J. F. Toole, "Endarterectomy for asymptomatic carotid artery stenosis," Journal of the American Medical Association, vol. 273, no. 18, pp. 1421-1428, 1995.

[6] A. Halliday, A. Mansfield, J. Marro et al., "Prevention of disabling and fatal strokes by successful carotid endarterectomy in patients without recent neurological symptoms: randomised controlled trial," Lancet, vol. 363, pp. 1491-1502, 2004.

[7] T. G. Brott, R. W. Hobson, G. Howard et al., "Stenting versus endarterectomy for treatment of carotid-artery stenosis," New England Journal of Medicine, vol. 363, no. 1, pp. 11-23, 2010.

[8] L. H. Bonati, J. Dobson, A. Algra et al., "Short-term outcome after stenting versus endarterectomy for symptomatic carotid stenosis: a preplanned meta-analysis of individual patient data," The Lancet, vol. 376, no. 9746, pp. 1062-1073, 2010.

[9] B. R. Chambers and J. W. Norris, "Outcome in patients with asymptomatic neck bruits," New England Journal of Medicine, vol. 315 , no. 14 , pp. $860-865,1986$. 
[10] M. Hennerici, H. B. Hülsbömer, H. Hefter et al., "Natural history of asymptomatic extracranial arterial disease. Results of a long-term prospective study," Brain, vol. 110, no. 3, pp. 777-791, 1987.

[11] M. E. DeBakey, "Successful carotid endarterectomy for cerebrovascular insufficiency. Nineteen-year follow-up," Journal of the American Medical Association, vol. 233, no. 10, pp. 10831085, 1975.

[12] H. H. Eastcott, G. W. Pickering, and C. G. Rob, "Reconstruction of internal carotid artery in a patient with intermittent attacks of hemiplegia," The Lancet, vol. 267, no. 6846, pp. 994996, 1954.

[13] A. Ois, E. Cuadrado-Godia, A. Rodríguez-Campello, J. Jimenez-Conde, and J. Roquer, "High risk of early neurological recurrence in symptomatic carotid stenosis," Stroke, vol. 40, no. 8, pp. 2727-2731, 2009.

[14] J. J. Badimon, B. Ibanez, and G. Cimmino, "Genesis and dynamics of atherosclerotic lesions: implications for early detection," Cerebrovascular Diseases, vol. 27, supplement 1, pp. 38-47, 2009.

[15] M. J. Davies, J. L. Gordon, A. J. Gearing et al., "The expression of the adhesion molecules ICAM-1, VCAM-1, PECAM, and E-selectin in human atherosclerosis," Journal of Pathology, vol. 171, no. 3, pp. 223-229, 1993.

[16] P. S. Gargalovic, M. Imura, B. Zhang et al., "Identification of inflammatory gene modules based on variations of human endothelial cell responses to oxidized lipids," Proceedings of the National Academy of Sciences of the United States of America, vol. 103, no. 34, pp. 12741-12746, 2006.

[17] L. Kerenyi, L. Mihalka, L. Csiba, H. Bacso, and D. Bereczki, "Role of hyperlipidemia in atherosclerotic plaque formation in the internal carotid artery," Journal of Clinical Ultrasound, vol. 34, no. 6, pp. 283-288, 2006.

[18] R. Ross, "The pathogenesis of atherosclerosis: a perspective for the 1990s," Nature, vol. 362, no. 6423, pp. 801-809, 1993.

[19] P. C. Schulze and R. T. Lee, "Oxidative stress and atherosclerosis," Current Atherosclerosis Reports, vol. 7, no. 3, pp. 242-248, 2005.

[20] F. D. Kolodgie, H. K. Gold, A. P. Burke et al., "Intraplaque hemorrhage and progression of coronary atheroma," New England Journal of Medicine, vol. 349, no. 24, pp. 2316-2325, 2003.

[21] P. Libby, "Inflammation in atherosclerosis," Nature, vol. 420, no. 6917, pp. 868-874, 2002.

[22] M. G. Hennerici and D. Neuerburg-Heussler, Vascular Diagnosis with Ultrasound, vol. 1, Georg Thieme, Stuttgart, Germany, 2006.

[23] M. G. Bousser, P. Amarenco, A. Chamorro et al., "Rationale and design of a randomized, double-blind, parallel-group study of terutroban $30 \mathrm{mg} /$ day versus aspirin $100 \mathrm{mg} /$ day in stroke patients: the prevention of cerebrovascular and cardiovascular events of ischemic origin with terutroban in patients with a history of ischemic stroke or transient ischemic attack (PERFORM) study," Cerebrovascular Diseases, vol. 27, no. 5, pp. 509-518, 2009.

[24] J. A. Chalela, "Evaluating the carotid plaque: going beyond stenosis," Cerebrovascular Diseases, vol. 27, supplement 1, pp. 19-24, 2009.

[25] S. Okazaki, S. Furukado, Y. Abe et al., "Association of inflammatory markers and carotid intima-media thickness with the risk of cardiovascular events in high-risk patients," Cerebrovascular Diseases, vol. 30, no. 2, pp. 180-187, 2010.
[26] T. Gao, Z. Zhang, W. Yu, Z. Zhang, and Y. Wang, "Atherosclerotic carotid vulnerable plaque and subsequent stroke: a highresolution MRI study," Cerebrovascular Diseases, vol. 27, no. 4, pp. 345-352, 2009.

[27] C. Enzinger, S. Ropele, T. Gattringer, C. Langkammer, R. Schmidt, and F. Fazekas, "High-grade internal carotid artery stenosis and chronic brain damage: a volumetric magnetic resonance imaging study," Cerebrovascular Diseases, vol. 30, no. 6, pp. 540-546, 2010.

[28] J. Golledge, R. Cuming, M. Ellis, A. H. Davies, and R. M. Greenhalgh, "Carotid plaque characteristics and presenting symptom," British Journal of Surgery, vol. 84, no. 12, pp. 16971701, 1997.

[29] R. Dittrich, M. A. Ritter, M. Kaps et al., "The use of embolic signal detection in multicenter trials to evaluate antiplatelet efficacy: signal analysis and quality control mechanisms in the CARESS (Clopidogrel and Aspirin for Reduction of Emboli in Symptomatic carotid Stenosis) trial," Stroke, vol. 37, no. 4, pp. 1065-1069, 2006.

[30] M. Daffertshofer, S. Ries, U. Schminke, and M. Hennerici, "High-intensity transient signals in patients with cerebral ischemia," Stroke, vol. 27, no. 10, pp. 1844-1849, 1996.

[31] M. G. Hennerici and S. Meairs, "Refined analysis of transcranial Doppler HITS," The Lancet Neurology, vol. 1, no. 7, p. 406, 2002.

[32] H. S. Markus, A. King, M. Shipley et al., "Asymptomatic embolisation for prediction of stroke in the Asymptomatic Carotid Emboli Study (ACES): a prospective observational study," The Lancet Neurology, vol. 9, no. 7, pp. 663-671, 2010.

[33] H. J. Barnett, D. W. Taylor, M. Eliasziw et al., "Benefit of carotid endarterectomy in patients with symptomatic moderate or severe stenosis. North American Symptomatic Carotid Endarterectomy Trial Collaborators," New England Journal of Medicine, vol. 339, no. 20, pp. 1415-1425, 1998.

[34] C. S. Cina, C. M. Clase, and R. B. Haynes, "Carotid endarterectomy for symptomatic carotid stenosis," Cochrane Database of Systematic Reviews, no. 2, p. CD001081, 2000.

[35] M. Paciaroni, M. Eliasziw, B. L. Sharpe et al., "Long-term clinical and angiographic outcomes in symptomatic patients with $70 \%$ to $99 \%$ carotid artery stenosis," Stroke, vol. 31, no. 9, pp. 2037-2042, 2000.

[36] P. M. Rothwell, S. A. Gutnikov, and C. P. Warlow, "Reanalysis of the final results of the European Carotid Surgery Trial," Stroke, vol. 34, no. 2, pp. 514-523, 2003.

[37] P. M. Rothwell, M. Eliasziw, S. A. Gutnikov et al., "Analysis of pooled data from the randomised controlled trials of endarterectomy for symptomatic carotid stenosis," Lancet, vol. 361, no. 9352, pp. 107-116, 2003.

[38] B. R. Chambers and G. A. Donnan, "Carotid endarterectomy for asymptomatic carotid stenosis," Cochrane Database of Systematic Reviews, no. 4, p. CD001923, 2005.

[39] A. Nicolaides, M. Sabetai, S. K. Kakkos et al., "The Asymptomatic Carotid Stenosis and Risk of Stroke (ACSRS) study. Aims and result of quality control," International Angiology, vol. 22, no. 3, pp. 263-272, 2003.

[40] Collaborators NASCET, "North American symptomatic carotid endarterectomy trial: methods, patient characteristics, and progress," Stroke, vol. 22, no. 6, pp. 711-720, 1991.

[41] H. J. Barnett, R. W. Gunton, M. Eliasziw et al., "Causes and severity of ischemic stroke in patients with internal carotid artery stenosis," Journal of the American Medical Association, vol. 283, no. 11, pp. 1429-1436, 2000. 
[42] P. Amarenco, J. Bogousslavsky, L. R. Caplan, G. A. Donnan, and M. G. Hennerici, "New approach to stroke subtyping: the A-S-C-O (phenotypic) classification of stroke," Cerebrovascular Diseases, vol. 27, no. 5, pp. 502-508, 2009.

[43] O. Honda, S. Sugiyama, K. Kugiyama et al., "Echolucent carotid plaques predict future coronary events in patients with coronary artery disease," Journal of the American College of Cardiology, vol. 43, no. 7, pp. 1177-1184, 2004.

[44] P. M. Rothwell, "Carotid artery disease and the risk of ischaemic stroke and coronary vascular events," Cerebrovascular Diseases, vol. 10, supplement 5, pp. 21-33, 2000.

[45] J. Y. Streifler, "Asymptomatic carotid stenosis: intervention or just stick to medical therapy-the case for medical therapy," Journal of Neural Transmission, vol. 118, no. 4, pp. 637-640, 2011.

[46] O. Lanczik, H. Bäzner, M. G. Hennerici, and R. Kern, "Asymptomatic carotid stenoses: course and discussion of their treatment," Nervenarzt, vol. 77, supplement 1, pp. S5S12, 2006.

[47] H. J. Barnett, H. E. Meldrum, and M. Eliasziw, "The appropriate use of carotid endarterectomy," CMAJ, vol. 166, no. 9, pp. 1169-1179, 2002.

[48] S. K. Kakkos, A. Nicolaides, M. Griffin et al., "Factors associated with mortality in patients with asymptomatic carotid stenosis: results from the ACSRS study," International Angiology, vol. 24, no. 3, pp. 221-230, 2005.

[49] S. K. Kakkos, M. Sabetai, T. Tegos et al., "Silent embolic infarcts on computed tomography brain scans and risk of ipsilateral hemispheric events in patients with asymptomatic internal carotid artery stenosis," Journal of Vascular Surgery, vol. 49, no. 4, pp. 902-909, 2009.

[50] S. Ries, U. Schminke, M. Daffertshofer, and M. Hennerici, "High intensity transient signals (HITS) in patients with carotid artery disease," European Journal of Medical Research, vol. 1, no. 7, pp. 328-330, 1996.

[51] P. M. Rothwell, "Prediction and prevention of stroke in patients with symptomatic carotid stenosis: the high-risk period and the high-risk patient," European Journal of Vascular and Endovascular Surgery, vol. 35, no. 3, pp. 255-263, 2008.

[52] H. J. Barnett, "The inappropriate use of carotid endarterectomy," CMAJ, vol. 171, no. 5, pp. 473-474, 2004.

[53] O. Benavente, M. Eliasziw, J. Y. Streifler, A. J. Fox, H. J. Barnett, and H. Meldrum, "Prognosis after transient monocular blindness associated with carotid-artery stenosis," New England Journal of Medicine, vol. 345, no. 15, pp. 1084-1090, 2001.

[54] P. M. Rothwell, "Risk modeling to identify patients with symptomatic carotid stenosis most at risk of stroke," Neurological Research, vol. 27, supplement 1, pp. S18-S28, 2005.

[55] B. L. Cucchiara, S. R. Messe, L. Sansing et al., "Lipoproteinassociated phospholipase A2 and C-reactive protein for riskstratification of patients with TIA," Stroke, vol. 40, no. 7, pp. 2332-2336, 2009.

[56] M. Eliasziw, J. Kennedy, M. D. Hill, A. M. Buchan, and H. J. Barnett, "Early risk of stroke after a transient ischemic attack in patients with internal carotid artery disease," CMAJ, vol. 170, no. 7, pp. 1105-1109, 2004.

[57] M. Eliasziw, J. Y. Streifler, A. J. Fox, V. C. Hachinski, G. G. Ferguson, and H. J. Barnett, "Significance of plaque ulceration in symptomatic patients with high-grade carotid stenosis. North American Symptomatic Carotid Endarterectomy Trial," Stroke, vol. 25, no. 2, pp. 304-308, 1994.

[58] T. S. Hatsukami, M. S. Ferguson, K. W. Beach et al., "Carotid plaque morphology and clinical events," Stroke, vol. 28, no. 1, pp. 95-100, 1997.
[59] M. G. Hennerici, “The unstable plaque," Cerebrovascular Diseases, vol. 17, supplement 3, pp. 17-22, 2004.

[60] S. C. Johnston, P. M. Rothwell, M. N. Nguyen-Huynh et al., "Validation and refinement of scores to predict very early stroke risk after transient ischaemic attack," Lancet, vol. 369, no. 9558, pp. 283-292, 2007.

[61] P. M. Rothwell, "Effective stroke prevention in patients with symptomatic carotid stenosis," Cerebrovascular Diseases, vol. 17, supplement 1, pp. 89-104, 2004.

[62] R. E. Petrea, A. S. Beiser, S. Seshadri, M. Kelly-Hayes, C. S. Kase, and P. A. Wolf, "Gender differences in stroke incidence and poststroke disability in the framingham heart study," Stroke, vol. 40, no. 4, pp. 1032-1037, 2009.

[63] D. J. Gladstone, J. Oh, J. Fang et al., "Urgency of carotid endarterectomy for secondary stroke prevention: results from the Registry of the Canadian Stroke Network," Stroke, vol. 40, no. 8, pp. 2776-2782, 2009.

[64] R. Kern, P. A. Ringleb, W. Hacke, J. L. Mas, and M. G. Hennerici, "Stenting for carotid artery stenosis," Nature Clinical Practice Neurology, vol. 3, no. 4, pp. 212-220, 2007.

[65] F. L. Silver, A. Mackey, W. M. Clark et al., "Safety of stenting and endarterectomy by symptomatic status in the Carotid Revascularization Endarterectomy Versus Stenting Trial (CREST)," Stroke, vol. 42, no. 3, pp. 675-680, 2011.

[66] P. Amarenco, L. B. Goldstein, A. Callahan 3rd et al., "Baseline blood pressure, low- and high-density lipoproteins, and triglycerides and the risk of vascular events in the Stroke Prevention by Aggressive Reduction in Cholesterol Levels (SPARCL) trial," Atherosclerosis, vol. 204, no. 2, pp. 515-520, 2009.

[67] H. Sillesen, P. Amarenco, M. G. Hennerici et al., "Atorvastatin reduces the risk of cardiovascular events in patients with carotid atherosclerosis: a secondary analysis of the stroke prevention by aggressive reduction in cholesterol levels (SPARCL) trial," Stroke, vol. 39, no. 12, pp. 3297-3302, 2008. 


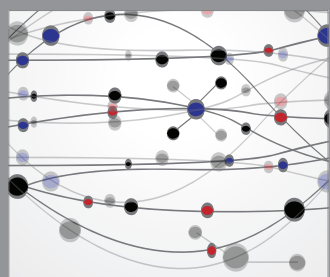

The Scientific World Journal
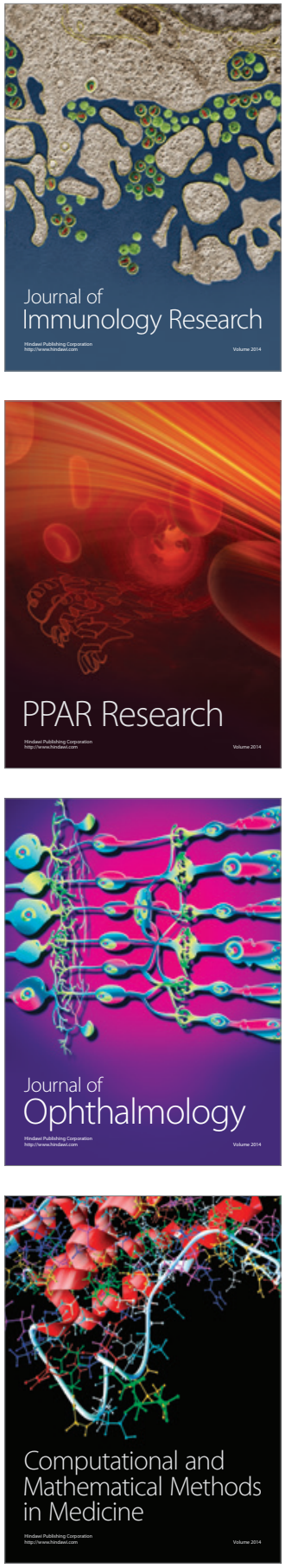

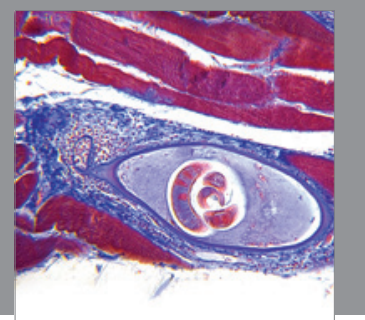

Gastroenterology

Research and Practice
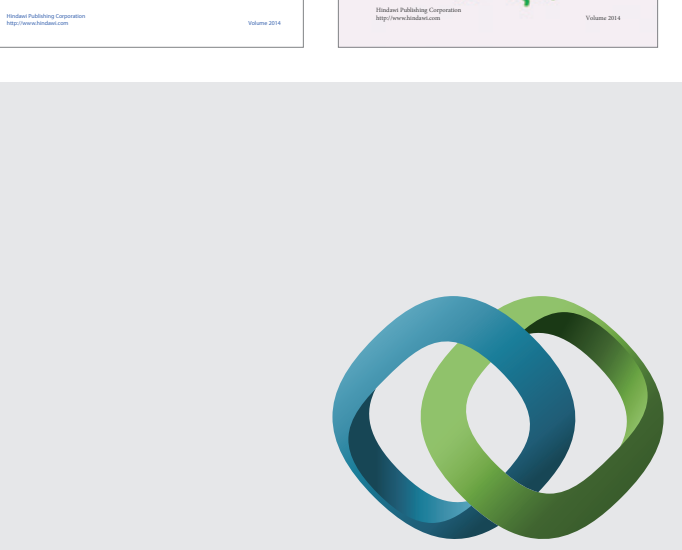

\section{Hindawi}

Submit your manuscripts at

http://www.hindawi.com
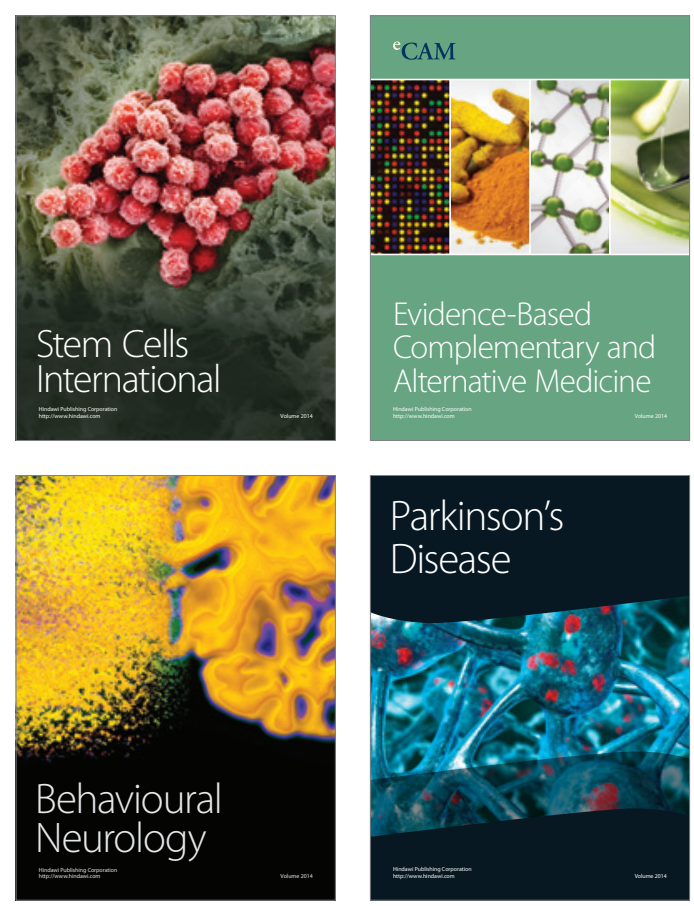

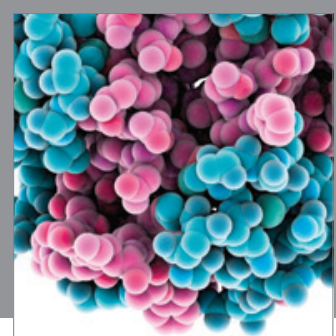

Journal of
Diabetes Research

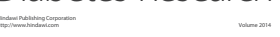

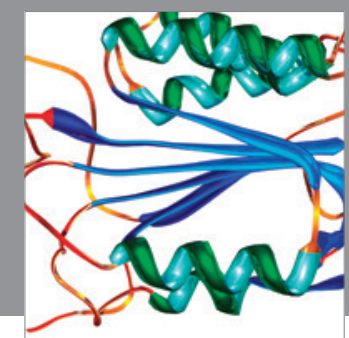

Disease Markers
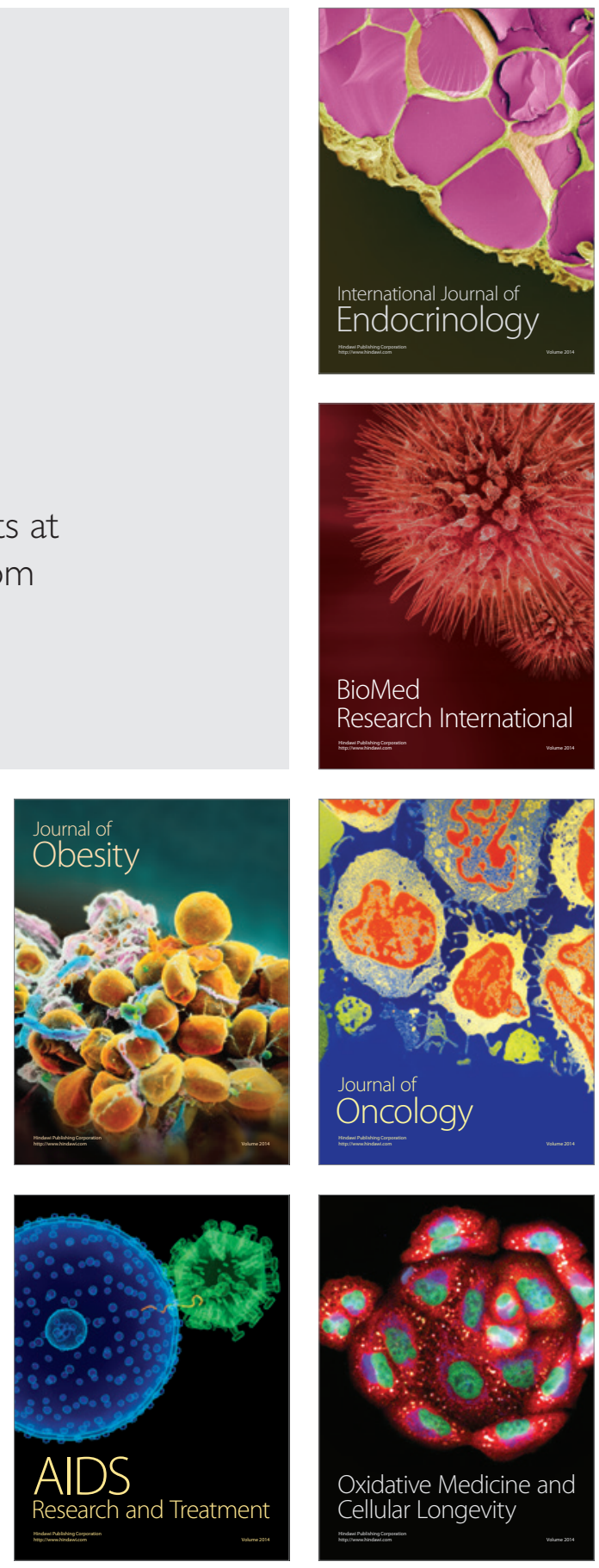